\title{
Cerebral Air Embolism: The Importance of Computed Tomography Evaluation
}

\author{
Catarina Brito ${ }^{\mathrm{a}, \mathrm{c}}$, Joana Graca ${ }^{\mathrm{b}}$, Pedro Vilela ${ }^{\mathrm{a}}$
}

\begin{abstract}
Cerebral air embolism (CAE) is a serious disease, often underdiagnosed due to nonspecific neurological findings and clinical and imaging unawareness. Early diagnosis is crucial for a prompt specific treatment, which differs from other types of brain infarcts. Immediate computed tomography (CT) scan is the imaging technique of choice, allowing to depict acute intracranial air, most commonly seen in the high convexity along cortical grooves, in a sulcal subarachnoid gyriform pattern. The use of thin-slice (thickness of $5 \mathrm{~mm}$ or smaller) and the measurement of air attenuation coefficient can reduce falsenegative studies. Hyperbaric oxygen therapy (HBOT) is the first-line treatment for CAE and may affect the outcome. We report four cases of iatrogenic $\mathrm{CAE}$ and review the literature.
\end{abstract}

Keywords: Cerebral air embolism; Central venous catheter; Hyperbaric oxygen therapy

\section{Introduction}

Vascular air embolism is a devastating event with high morbidity and mortality. Iatrogenic injury is the most frequent cause, associated with several medical-surgical procedures. There is an increase in its incidence in the last decades in parallel with technological advances in the medical field [1-4].

Neurological findings of cerebral air embolism (CAE) are nonspecific and include an altered state of consciousness, seizures, and stroke-like symptoms or may even be clinically silent. Systemic manifestations can also be present, such as respiratory distress, hemodynamic instability, arrhythmia, and cardio-respiratory arrest $[4,5]$.

When facing a suspected CAE, an immediate computed tomography (CT) scan is essential for early diagnosis and man-

Manuscript submitted September 12, 2020, accepted September 22, 2020

Published online October 21, 2020

aDepartment of Neuroradiology, Hospital Beatriz Angelo, Loures, Portugal bDepartment of Neuroradiology, Hospital Egas Moniz, Lisbon, Portugal ${ }^{\mathrm{c} C o r r e s p o n d i n g ~ A u t h o r: ~ C a t a r i n a ~ B r i t o, ~ D e p a r t m e n t ~ o f ~ N e u r o r a d i o l o g y, ~ H o s-~}$ pital Beatriz Angelo, Avenida Carlos Teixeira 3, Loures 2674-514, Portugal. Email: catarinaborgesbrito@gmail.com

doi: https://doi.org/10.14740/jmc3583 agement, since specific treatment may affect the outcome [6].

We report four cases of CAE diagnosed by brain CT scan, three after removal of a central venous catheter (CVC) (one for hemodialysis and two for chemotherapy) and one after endoscopic retrograde cholangiopancreatography (ERCP).

\section{Case Reports}

\section{Case 1}

A 65-year-old male patient with HIV under antiretroviral therapy was admitted with acute renal failure, requiring hemodialysis. After three sessions, the CVC was removed and the patient had sudden altered level of consciousness with Glasgow Coma Scale (GCS) 6, deviation of the gaze to the right and bilateral extensor plantar response.

Emergency brain CT scan (Fig.1a) revealed multiple bihemispheric frontoparietal curvilinear hypodensities consistent with air in the cortical sulci, distributed predominantly in the right hemisphere. Unfortunately, these findings were only adequately valued after image review, which led to the late etiological diagnosis.

Follow-up brain magnetic resonance imaging (MRI) (Fig. 1b) (48 $\mathrm{h}$ after the onset) showed large right frontoparietal cortical ischemic lesions, with additional small left perirolandic cortex involvement. No MRI signal abnormality was identified at the subarachnoid space and CT scan at the same time demonstrated resorption of the air content located in the subarachnoid/intravascular space. These imaging findings were suggestive of CAE involving multiple arterial territories with associated extensive laminar cortical necrosis.

Hyperbaric oxygen therapy (HBOT) was not offered during the acute phase since the diagnosis was not promptly performed. The patient had a slight neurological improvement, presenting at the time of discharge, left hemiparesis (muscle strength grade $4 / 5$ ).

\section{Case 2}

A 36-year-old female with human immunodeficiency viruses type 1 (HIV-1) stage 3 under chemotherapy for Castleman's disease was admitted for suspected infective endocarditis. After CVC removal, the patient presented a simple partial seizure with secondary generalization, altered level of consciousness 


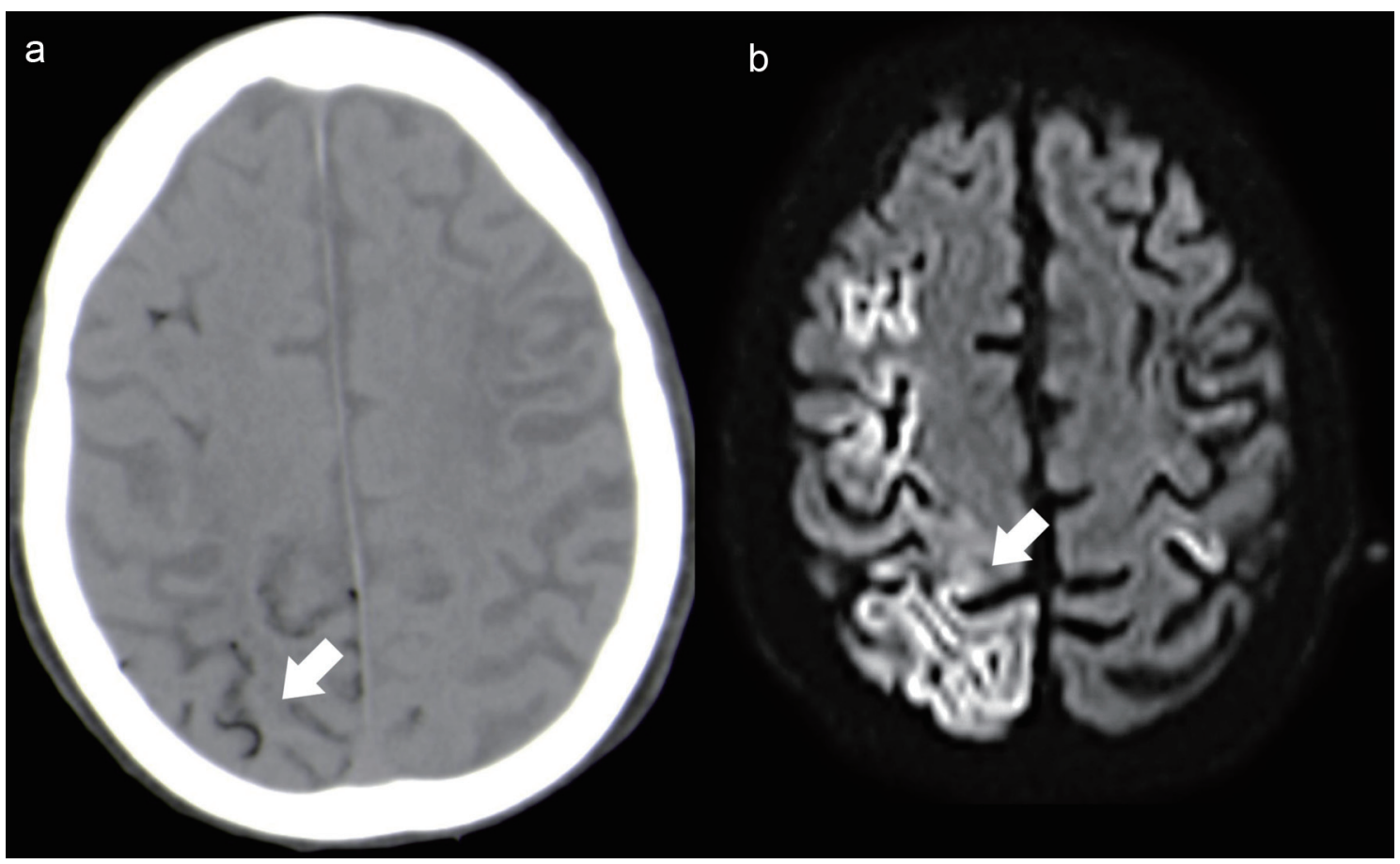

Figure 1. Case 1. Axial NCCT (a) depicts multiple small hypodense foci located mainly in the right cortical sulci (white arrow). Follow-up brain MRI (48 $\mathrm{h}$ after the onset), axial DWI (b) shows extensive right frontoparietal cortical ischemic lesions (white arrow), with additional small left perirolandic cortex involvement. NCCT: non-contrast computed tomography; MRI: magnetic resonance imaging; DWI: diffusion-weighted imaging.

and subsequent left hemiparesis (muscle strength grade 3/5).

Emergency CT scan demonstrated multiple bilateral micro-hypodensities distributed in the subarachnoid space along high cerebral convexity. Unfortunately, these findings were only adequately valued after image review, which led to the late etiological diagnosis.

Follow-up CT scan (4 days after the onset) showed disappearance of the hypodense content in the subarachnoid/ vascular space and presence of cerebral infarctions with the same topographic distribution.

HBOT was not offered during the acute phase since the diagnosis was not promptly performed. At the time of hospital discharge, the patient did not present significant neurological improvement, remaining with left hemiparesis (muscle strength grade $3 / 5$ ).

\section{Case 3}

A 57-year-old man diagnosed with sigmoid adenocarcinoma and undergoing chemotherapy, was admitted for resection of a left cerebellar hemisphere metastasis. Three days after surgery, CVC was removed and the patient experienced sudden onset of involuntary movements and altered level of consciousness, followed by left hemiparesis (muscle strength grade 3/5).

Emergency brain CT scan (Fig. 2a) was performed and showed intracranial air with vascular distribution. These findings were only assessed during image review on the following day, which led to a diagnostic delay.
Follow-up MRI scan (Fig. 2c, d), performed 3 days after the onset documented cortical ischemia and parenchymal ede$\mathrm{ma}$, with the same distribution of the intracranial air, no longer documented on the follow-up CT scan (Fig. 2b).

HBOT was not offered during the acute phase since the diagnosis was not promptly performed. The patient underwent physiotherapy with slow improvement, presenting at the time of discharge muscle strength grade $4 / 5$ in the left limbs.

\section{Case 4}

An 89-year-old man diagnosed with cholangiocarcinoma was submitted to endoscopic retrograde cholangiopancreatography with endoscopic endoprosthesis insertion. After endoscope removal, the patient had sudden desaturation, bradycardia and altered level of consciousness (GCS 3) with further need for cardiopulmonary resuscitation.

Emergency CT scan showed diffuse subarachnoid intracranial air (Fig. 3a, b), suggestive of gas embolism and imaging findings indicative of hypoxic-ischemic encephalopathy (Fig. 3c, d).

The presence of large ischemic lesions on CT and the dismal patient prognosis precluded additional treatment actions and HBOT was not suggested. The patient died $24 \mathrm{~h}$ after the onset.

\section{Discussion}

Vascular air embolism is a rare event, initially described in 


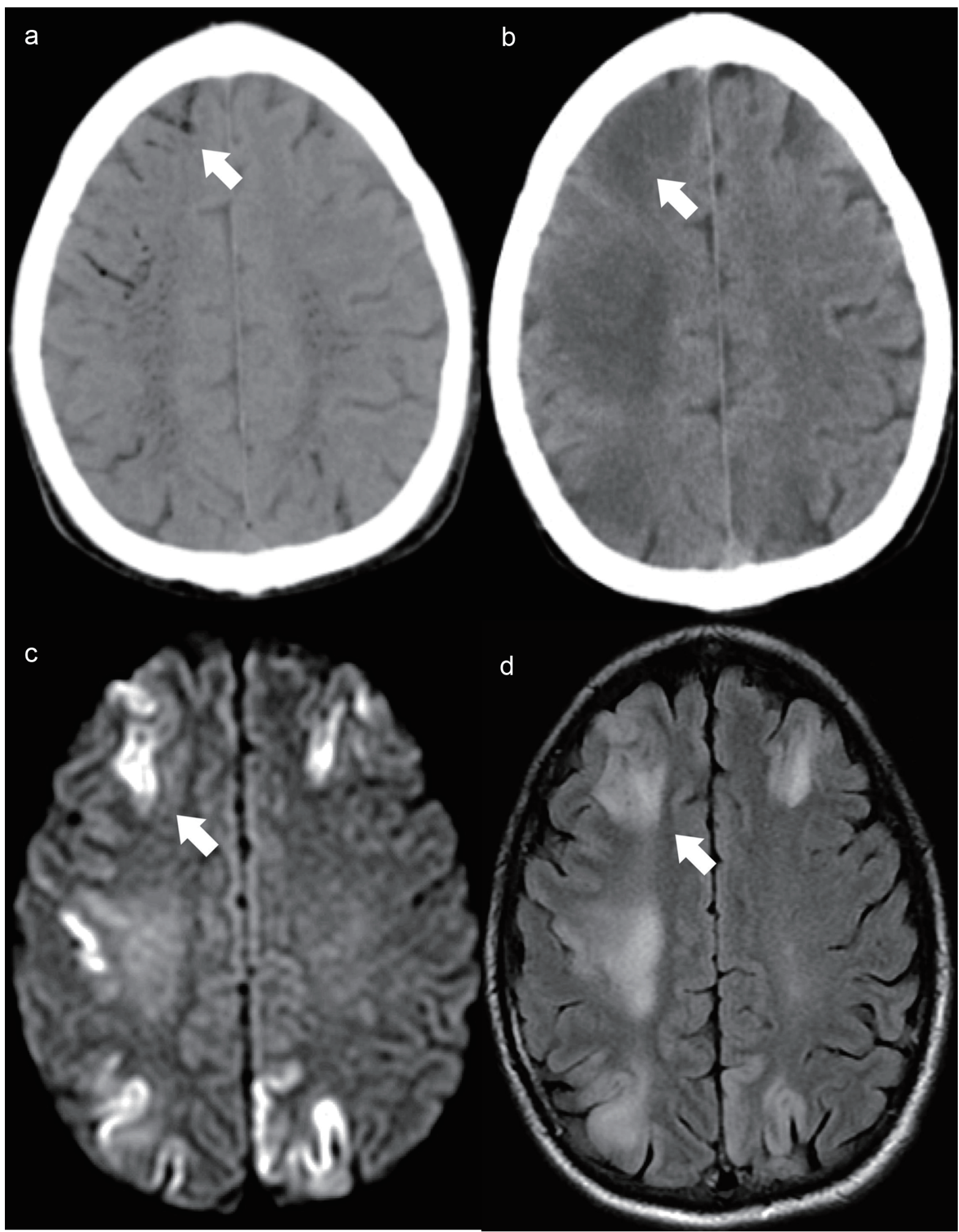

Figure 2. Case 3. Brain axial NCCT (a) demonstrates filiform air hypodensities in the high convexity sulci (white arrow) and intraparenchymal with a vascular distribution, predominantly in the semioval center. Control brain axial NCCT scan (b) reveals intracranial air reabsorption and frontoparietal cortico-subcortical dedifferentiation (white arrow). Brain MRI axial DWI (c) shows bilateral fronto-parietal cortical diffusion restriction with the corresponding cortical hyperintensity on axial FLAIR image (d) and evidence of white matter vasogenic edema (white arrow). NCCT: non-contrast computed tomography; MRI: magnetic resonance imaging; DWI: diffusion-weighted imaging; FLAIR: fluid-attenuated inversion recovery. 


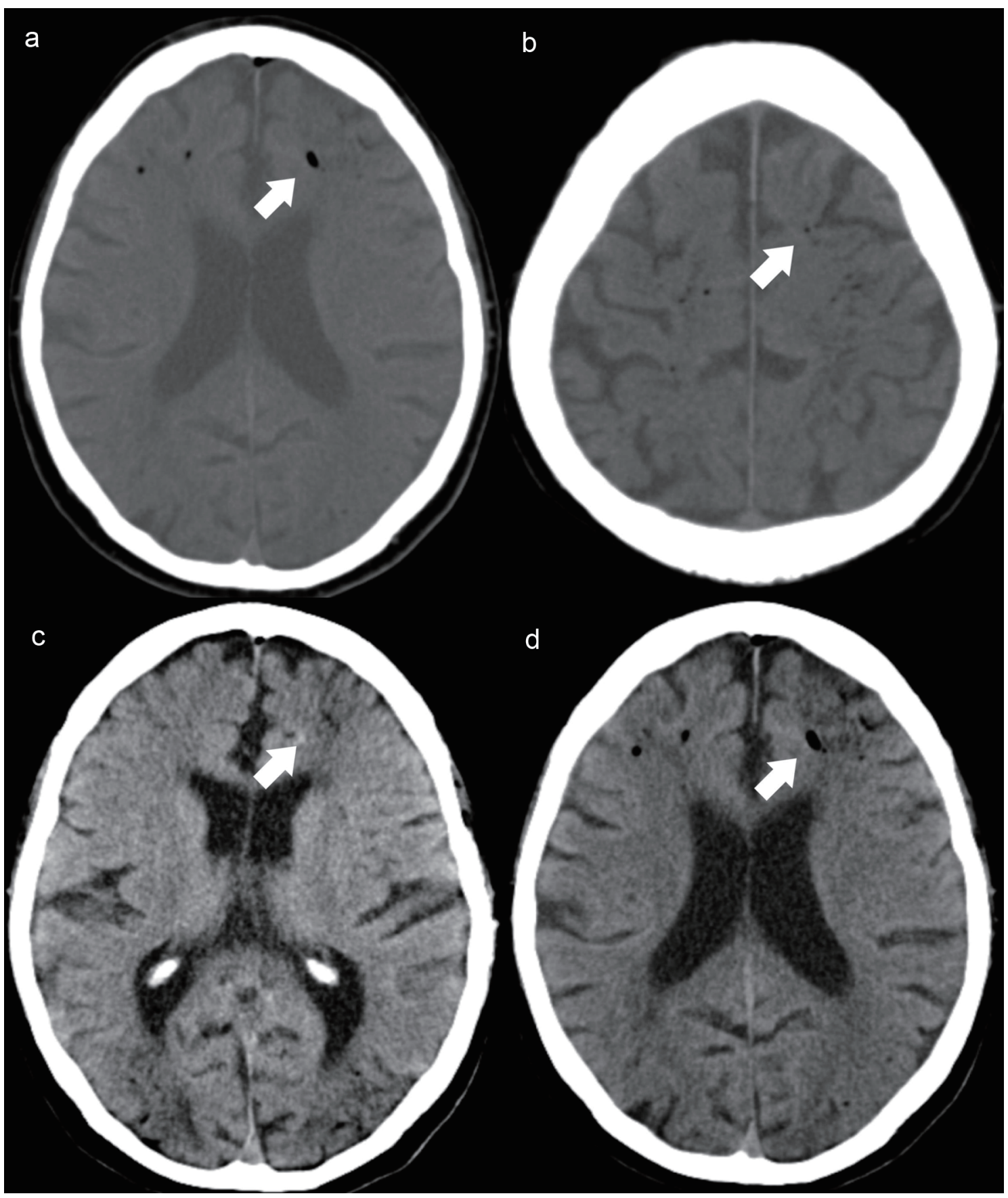

Figure 3. Case 4. Axial NCCT scan (a, b) demonstrates intracranial air, along the frontal cortical sulci (white arrows) and adjacent hypodense areas in frontal and parieto-occipital regions, revealing watershed infarcts (c, d) hypoxic-ischemic encephalopathy (white arrows). NCCT: non-contrast computed tomography.

the 19th century, associated with medical-surgical procedures, penetrating injuries and barotrauma. The number of clinical reports has been increasing in recent years, along with the expansion of invasive medical and surgical procedures and therapeutic methods [7]. The real incidence is unknown, since misdiagnoses are common. For CVC and cardiothoracic surgery with cardiac bypass, the incidence is estimated to be $0.13 \%$ and $0.003-0.007 \%$, respectively [8], with frequent intracranial involvement. The epidemiological studies regarding specifically to CAE are limited [9]. It is often associated with CVC management or neurosurgical and otorhinolaryngological procedures.

Vascular gas embolism implies a negative pressure gradient and direct communication between the vascular system and atmospheric air. Both venous and arterial intracranial air have been associated with $\mathrm{CAE}$, with recent reports arguing 
that cerebral arterial air is required to produce neurological complications $[4,5,9]$.

CAE may occur in the presence of an intra-cardiac or intra-pulmonary shunt, and/or when multiple air emboli, that are small enough to be filtered through the pulmonary vasculature, are embolized through the systemic circulation (paradoxical embolism). Vasodilators or anaesthetic agents may enhance the later mechanism. Once in the intracranial arterial circulation, the gas bubbles preferentially occlude small caliber arteries (mean diameter 30 to $60 \mu \mathrm{m}$ ) [5] causing brain injury by reduction of cerebral parenchyma perfusion distal to the occlusion, in terminal arterial territories, and/or by a local inflammatory reaction activated by the presence of air [10-12].

Cerebral arterial gas embolism has a predilection for supratentorial compartment with bilateral distribution, but involving mainly the right cerebral hemisphere, since the brachiocephalic trunk is the major first branch of the aorta. Distribution of intracranial air may be related to patient's position at the time of the event, with the prone position causing emboli located mostly in the posterior circulation due to preferential flow to the right vertebral artery [13].

It has been suggested that $\mathrm{CVC}$ associated $\mathrm{CAE}$ with no documented right-left shunt could result from passage of venous emboli by a retrograde venous movement to the jugular veins, especially if the patient is seated with high back [14, $15]$.

The grade of morbidity and mortality in vascular gas embolism is associated with gas volume, accumulation rate, and patient position at the time of the embolic event. In CAE, the topography where air bubbles accumulate is of paramount importance to predict the outcome, and paradoxically a small amount of air can have catastrophic consequences [4].

There are no specific neurological findings for CAE, and it may even be clinically silent. As any ischemic stroke, its neurological manifestations depend on the brain region affected, frequently manifesting with stroke-like symptoms and accompanied by changes in the level of consciousness and general seizures. The suspicion must be raised in the presence of systemic symptoms, such as respiratory distress, arrhythmias and cardiorespiratory arrest. The mill-wheel murmur indicates the presence of intracardiac air but is only present in one-third of patients [1].

Patients with suspected CAE should undergo emergent CT scan, to identify the presence of intracranial air within the first hour from the onset, owing to rapid resorption of air by brain arterioles. It should be emphasised that late examination may not show the presence of air [1]. This might explain the underrecognition of this disease. Non-contrast CT scan is a highly sensitive imaging technique to detect the presence of small amounts of air. It allows direct visualization of air emboli, such as small round or curvilinear hypodensities (gyriform pattern), representing air in the cerebral cortical vessels [9].

Potential causes of false-negative studies and reduced CT scan imaging sensitivity include non-visualization of small amounts of air, gas reabsorption before acquisition, misinterpretation of air attenuation coefficient as fat [13]. The use of thin-slice thickness ( $5 \mathrm{~mm}$ or smaller) and air attenuation coefficient measurement, using the lung window settings and comparing with the attenuation coefficient of the paranasal sinuses, increase the diagnostic accuracy. Although rarely reported, gas emboli are also visible in MRI gradient echo sequences due to their susceptibility artifacts $[13,16]$.

The diagnosis of CAE by MRI is challenging. The imaging findings are non-specific and include cortical diffusion restriction (cytotoxic edema) areas. Additionally, adjacent areas of vasogenic edema may also be present. These features are similar to those documented in our cases, depicted by cortical necrosis due to acute local ischemia and vasogenic edema as a result of regional inflammatory reaction. CT and MRI perfusion studies in these patients are scarce, often presenting nonspecific findings. In CAE the whole brain CT perfusion may demonstrate areas of ischemia or penumbra in the region where the air bubbles are lodged, findings that are similar to those seen in acute ischemic lesions [17].

Recently, studies have addressed the prognostic value of neuroimaging evaluation in these patients, and the presence of intracranial air in a gyriform pattern could be an indicator of unfavourable outcome [18]. Fatal cases are usually associated with the coexistence of intracardiac air or with massive CAE $[19,20]$.

Early detection of CAE promotes proper management and treatment, including immediate airway closing, positioning in Trendelenburg, adequate oxygenation, in addition to other support measures, as well as HBOT [4-7, 21].

HBOT is the first-line treatment for CAE and should be initiated soon after the embolic event, within the first $4-6 \mathrm{~h}$ after symptoms onset, to ensure a good prognosis. It has been suggested that the beneficial role of HBOT can be extended up to $30 \mathrm{~h}$ after the initial event [6].

HBOT induces a reduction in air bubble size by increasing the ambient pressure and reduces the amount of air by increasing systemic hyperoxygenation. The parenchymal edema may also be reduced, by promoting the integrity of the blood-brain barrier and by decreasing leukocytes adhesion to the endothelium.

\section{Conclusions}

CAE is often underdiagnosed despite its potential devastating consequences. The suspicion must be raised in the appropriate clinical setting for high-risk patients. Brain CT scan is the imaging technique of choice and should be immediately performed, due to its high sensitivity for gas detection. MRI cannot generally depict CEA. It is essential to be aware of this severe disease to be able to make an early diagnosis, which may significantly impact patients' management and outcome.

\section{Acknowledgments}

The authors would like to acknowledge J. Barreira who contributed to clinical patient information, study design and image selection.

\section{Financial Disclosure}

None to declare. 


\section{Conflict of Interest}

None to declare.

\section{Informed Consent}

Not applicable.

\section{Author Contributions}

Catarina Brito collected data and wrote the manuscript. Joana Graca and Pedro Vilela were the final draft reviewers.

\section{Data Availability}

The authors declare that data supporting the findings of this study are available within the article.

\section{References}

1. Jensen ME, Lipper MH. CT in iatrogenic cerebral air embolism. AJNR Am J Neuroradiol. 1986;7(5):823-827.

2. Trabanco S, Pardo S, Williams M, Diaz J, Ruiz C. Cerebral air embolism after ERCP. J Clin Anesth. 2017;36:133135.

3. Finsterer J, Stollberger C, Bastovansky A. Cardiac and cerebral air embolism from endoscopic retrograde cholangio-pancreatography. Eur J Gastroenterol Hepatol. 2010;22(10):1157-1162.

4. Brull SJ, Prielipp RC. Vascular air embolism: A silent hazard to patient safety. J Crit Care. 2017;42:255-263.

5. Muth CM, Shank ES. Gas embolism. N Engl J Med. 2000;342(7):476-482.

6. McCarthy CJ, Behravesh S, Naidu SG, Oklu R. Air Embolism: Diagnosis, Clinical Management and Outcomes. Diagnostics (Basel). 2017;7(1).

7. Shaikh N, Ummunisa F. Acute management of vascular air embolism. J Emerg Trauma Shock. 2009;2(3):180185.

8. Gordy S, Rowell S. Vascular air embolism. Int J Crit Illn
Inj Sci. 2013;3(1):73-76.

9. Pinho J, Amorim JM, Araujo JM, Vilaca H, Ribeiro M, Pereira J, Ferreira C. Cerebral gas embolism associated with central venous catheter: Systematic review. J Neurol Sci. 2016;362:160-164.

10. Dutka AJ. A review of the pathophysiology and potential application of experimental therapies for cerebral ischemia to the treatment of cerebral arterial gas embolism. Undersea Biomed Res. 1985;12(4):403-421.

11. Moon RE. Hyperbaric oxygen treatment for air or gas embolism. Undersea Hyperb Med. 2014;41(2):159-166.

12. Helps SC, Gorman DF. Air embolism of the brain in rabbits pretreated with mechlorethamine. Stroke. 1991;22(3):351-354.

13. Zakhari N, Castillo M, Torres C. Unusual cerebral emboli. Neuroimaging Clin N Am. 2016;26(1):147-163.

14. Eum da H, Lee SH, Kim HW, Jung MJ, Lee JG. Cerebral air embolism following the removal of a central venous catheter in the absence of intracardiac rightto-left shunting: a case report. Medicine (Baltimore). 2015;94(13):e630.

15. Pellise A, Ustrell X, Ruiz V, Guedea A. [Retrograde venous cerebral air embolism as a cause of stroke]. Neurologia. 2012;27(2):119-121.

16. Jeon SB, Kang DW. Cerebral air emboli on T2-weighted gradient-echo magnetic resonance imaging. BMJ Case Rep. 2009;2009:bcr2006102954.

17. Murphy A, Torres C, Lum C, Hogan M, Bussiere M. Whole brain CT Perfusion after cerebral air embolism. Can J Neurol Sci. 2011;38(3):522-525.

18. Cheng CK, Chang TY, Liu CH, Chang $\mathrm{CH}$, Huang KL, $\mathrm{Chin} \mathrm{SC}, \mathrm{Wu} \mathrm{HC}$, et al. Presence of gyriform air predicts unfavorable outcome in venous catheter-related cerebral air embolism. J Stroke Cerebrovasc Dis. 2015;24(10):2189-2195.

19. Villanueva C, Manganas C. Fatal cerebral air embolism following central venous catheter removal. Hear Lung Circ. 2015;24:e40.

20. Andrade CS, Lucato LT, da Costa Leite C. Neurological picture. Massive fatal cerebral air embolism as a negative contrast angiogram. J Neurol Neurosurg Psychiatry. 2008;79(12):1357-1358.

21. McCarthy CJ, Behravesh S, Naidu SG, Oklu R. Air embolism: practical tips for prevention and treatment. J Clin Med. 2016;5(11). 